\title{
Bacteria of the Burkholderia cepacia complex are cyanogenic under biofilm and colonial growth conditions
}

\author{
Ben Ryall, Xiaoyun Lee, James EA Zlosnik, Saiko Hoshino and \\ Huw D Williams*
}

\begin{abstract}
Address: Department of Life Sciences, Division of Biology, Faculty of Natural Sciences, Imperial College London, Sir Alexander Fleming Building London, SW7 2AZ, UK

Email: Ben Ryall - ben.ryall00@imperial.ac.uk; Xiaoyun Lee - Xiaoyun.Lee@unil.ch; James EA Zlosnik - zlosnikjea@gmail.com; Saiko Hoshino - Saiko.Hoshino@morganstanley.com; Huw D Williams* - h.d.williams@imperial.ac.uk

* Corresponding author
\end{abstract}

Published: 27 June 2008

BMC Microbiology 2008, 8:108 doi:10.1186/147/-2180-8-108
Received: II December 2007

Accepted: 27 June 2008

This article is available from: http://www.biomedcentral.com/I47I-2/80/8//08

(c) 2008 Ryall et al; licensee BioMed Central Ltd.

This is an Open Access article distributed under the terms of the Creative Commons Attribution License (http://creativecommons.org/licenses/by/2.0), which permits unrestricted use, distribution, and reproduction in any medium, provided the original work is properly cited.

\begin{abstract}
Background: The Burkholderia cepacia complex $(\mathrm{Bcc})$ is a collection of nine genotypically distinct but phenotypically similar species. They show wide ecological diversity and include species that are used for promoting plant growth and bio-control as well species that are opportunistic pathogens of vulnerable patients. Over recent years the Bcc have emerged as problematic pathogens of the CF lung. Pseudomonas aeruginosa is another important CF pathogen. It is able to synthesise hydrogen cyanide $(\mathrm{HCN})$, a potent inhibitor of cellular respiration. We have recently shown that $\mathrm{HCN}$ production by $P$. aeruginosa may have a role in CF pathogenesis. This paper describes an investigation of the ability of bacteria of the Bcc to make HCN.
\end{abstract}

Results: The genome of Burkholderia cenocepacia has 3 putative $\mathrm{HCN}$ synthase encoding (hcnABC) gene clusters. B. cenocepacia and all 9 species of the Bcc complex tested were able to make cyanide at comparable levels to $P$. aeruginosa, but only when grown surface attached as colonies or during biofilm growth on glass beads. In contrast to $P$. aeruginosa and other cyanogenic bacteria, cyanide was not detected during planktonic growth of Bcc strains.

Conclusion: All species in the Bcc are cyanogenic when grown as surface attached colonies or as biofilms.

\section{Background}

The genus Burkholderia is a group of Gram-negative, nonspore forming $\beta$-proteobacteria with huge metabolic potential and wide ecological diversity [1]. The Burkholderia genus comprises more than 40 species, which range from plant symbionts to commercially important rice pathogens and from opportunistic pathogens of humans to the deadly and potential bio-warfare agent $B$. pseudomallei $[1,2]$.
The Burkholderia cepacia complex (Bcc) is a collection of nine genotypically distinct but phenotypically similar species within the Burkholderia genus [3,4]. The origins of the Bcc stem from a seminal study by Vandamme et al. [5] when 128 B. cepacia strains were analysed by genetic and phenotypic means and found to fall into at least five genomic species or genomovars. Over the coming years four other genomovars were assigned to the Bcc complex and each genomovar has now been assigned with a dis- 
tinct species name, which has replaced the use of the genomovar nomenclature $[3,4]$.

The Bcc show substantial metabolic diversity, and have a relatively unusual (for bacteria) multireplicon genomic arrangement, with all species having more than one chromosome and large genomes ranging from 6 to $9 \mathrm{Mb}[4]$. The Bcc show wide ecological diversity and include species that are used for promoting plant growth and biocontrol as well species that are opportunistic pathogens of vulnerable patients $[1,4]$.

Over recent years the Bcc have emerged as problematic pathogens of the CF lung. All of the species in the Bcc have been isolated from CF lung infections, but the most prominent is B. cenocepacia, which accounts for around $70 \%$ of all Bcc infections of the CF lung [4,6,7]. CF patients usually acquire Bcc infections later in the course of the disease, often once they are already chronically colonised by $P$. aeruginosa [7]. Similar to P. aeruginosa, Bcc strains can form chronic, persistent $\mathrm{CF}$ lung infections which are intractable to antibiotic therapy $[6,7]$. However, in contrast to $P$. aeruginosa, infection can vary from the fairly benign to a rapid respiratory failure and death, referred to as "cepacia syndrome" $[8,9]$. The diverse pathogenicity in CF appears to be strain rather than species specific with patient factors probably also playing a part since variability is seen in clinical outcome between patients infected with the same strain [4]. Bcc strains possess a number of potential virulence factors that may play a role in CF infection including; LPS, extracellular protease and the ability to form biofilms $[4,10]$. Similar to $P$. aeruginosa, most of these factors are under the control of quorum sensing, with all species in the Bcc possessing an $\mathrm{N}$-acyl-homoserine lactone signal molecule based system known as the CepI/CepR quorum sensing system $[11,12]$.

B. cenocepacia and other members of the Bcc are able to cocolonise the CF lung with $P$. aeruginosa $[6,7]$. We have recently shown that $P$. aeruginosa infection of the CF lung is associated with the accumulation of cyanide at a mean concentration of $72 \mu \mathrm{M}$ in the sputum, which correlates with a decline in lung function in CF patients [13]. Under these conditions of cyanide accumulation in the sputum Bcc can co-colonise with $P$. aeruginosa. Therefore, B. cenocepacia may also be resistant to the toxic effects of cyanide. Indeed high level cyanide tolerance has been reported in a B. cepacia strain [14]. One reason why an organism may be tolerant to cyanide is that it is itself capable of producing cyanide.

The aim of this work was to determine whether Bcc complex bacteria are able to make cyanide.

\section{Results \\ B. cenocepacia has three potential homologues of the P. aeruginosa cyanide synthase}

Cyanide production in $P$. aeruginosa requires the hydrogen cyanide synthase enzyme complex encoded by the $h c n A B C$ gene locus. We searched the genome sequence of B. cenocepacia J2315 [15] to see if the bacterium possessed genes that could potentially code for a cyanide synthase. A TBLASTN search of the B. cenocepacia genome using the sequence of the P. aeruginosa HcnA, HcnB and HcnC proteins [16] revealed that there were three possible homologues of the $P$. aeruginosa cyanide synthase in $B$. cenocepacia, two on chromosome 2 and one on chromosome 3, the organisation of which are shown in Fig. 1A. Several motifs are believed to be important to hydrogen cyanide synthase function [17]. In HcnA there is a motif containing four cysteine residues believed to be a binding site for an Fe-S cluster, in HcnB and HcnC there is an eleven amino acid motif defined as an ADP-binding motif [17]. These motifs are conserved in each of the three putative HCN synthase clusters in J2315. (Figure 1B, C and 1D)

\section{Cyanide is not detected in liquid cultures of $B$. cenocepacia strains}

We looked for cyanide production by B. cenocepacia K562 and $\mathrm{J} 2315$ growing in LB medium, sampling throughout exponential growth and up to $35 \mathrm{~h}$ into stationary phase. Cyanide was not detected in any samples during growth at $30^{\circ} \mathrm{C}$ or $37^{\circ} \mathrm{C}$. The $\mathrm{pH}$ of the culture media was measured and was always around $\mathrm{pH} 8$, which is the same as that seen for $P$. aeruginosa cultures in which cyanide is readily detected [18]. This suggests that cyanide was not being lost from the culture as hydrogen cyanide gas due to acidification of the culture medium. Glycine is the precursor for hydrogen cyanide synthesis in $P$. aeruginos $a$ and addition of glycine to medium stimulates $P$. aeruginosa cyanide production; methionine also enhances cyanide production $[19,20]$. However, no cyanide was detected when $B$. cenocepacia K56-2 and J2315 were grown in LB broth + glycine $(12.5 \mathrm{mM})$ and/or methionine $(5 \mathrm{mM})$. Oxygen is a regulator of cyanide synthesis in $P$. aeruginosa, with synthesis being promoted at low oxygen levels, and so we looked at whether varying oxygen availability led to cyanide formation in liquid culture [17]. However, despite varying the $\mathrm{O}_{2}$-transfer coefficient $\left(\mathrm{k}_{\mathrm{L}} \mathrm{a}\right)$ values from 87.4 $\mathrm{h}^{-1}$ (high) to $11.5 \mathrm{~h}^{-1}$ (low), no cyanide was detected at any stage of growth [21] (data not shown). We conclude that planktonic cultures of B. cenocepacia strains K56-2 and J2315 did not accumulate cyanide at detectable levels, which is in contrast to $P$. aeruginosa which accumulates cyanide to a concentration of 300-500 $\mu \mathrm{M}$ under identical growth conditions $[19,22,23]$. 
A

\section{$P$. aeruginosa henABC locus}

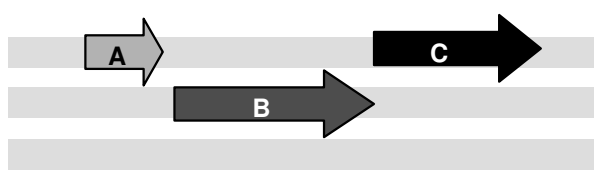

B. cenocepacia chromosome 2 BCAM0667-9

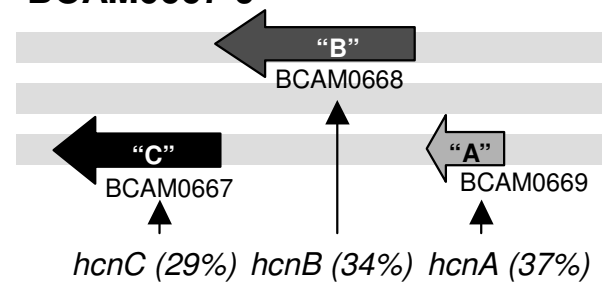

\section{B. cenocepacia chromosome 2 BCAM1268-9}

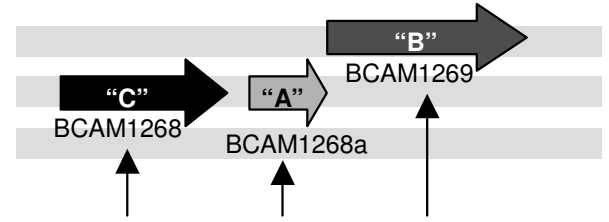

hcnC (30\%) hcnA (45\%) hcnB (32\%)

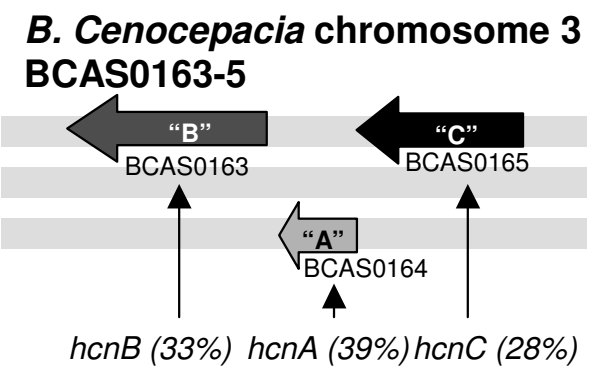

B

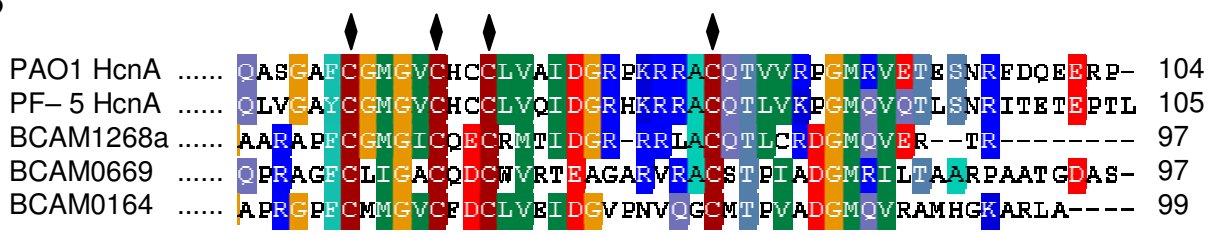

C

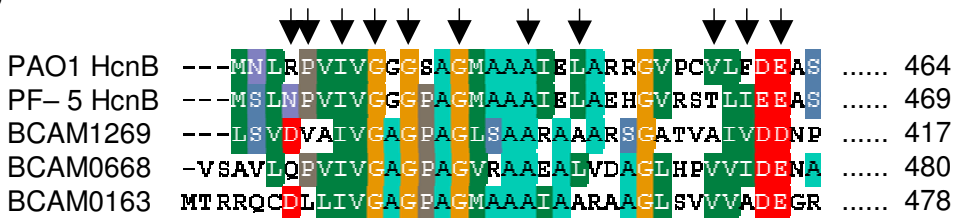

D

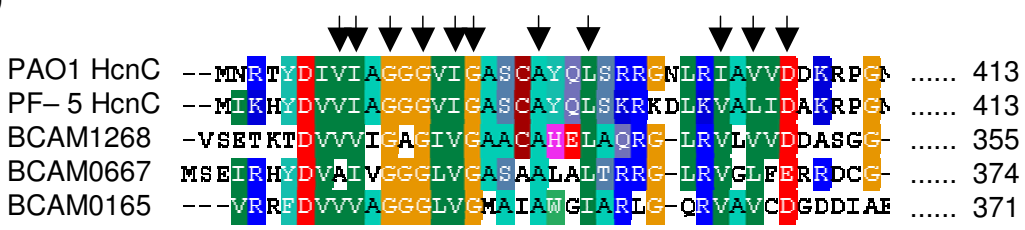

\section{Figure I}

Putative henABC genes in $B$. cenocepacia. A. The $P$. aeruginosa cyanide synthase enzyme complex is coded for by the henABC gene locus. $B$. cenocepacia has three sets of possible homologues of the $P$. aerugin $h a n A B C$ genes. The passible homologues of the $P$. aeruginosa hcnABC genes. The organisation of the $P$. aeruginosa hcnABC locus is shown in the top left panel followed by the three sets of $B$. cenoce-2 and bottom +/- 3; the arrows indicate if the genes are on the coding (left to right) or complement (right to left) strands. The percentage identity of the product of each putative gene to their $P$. aeruginosa HcnABChomologue is shown in labels underneath each panel. The Putative Bcc cyanide synthase proteins have conserved Fe-S (HcnA) and ADP binding motifs $(\mathrm{HcnB}$ and $\mathrm{HcnC}$ ). B. C. and D. ClustalX alignments of putative $B$ cenocepacia 12315 cyanide synthase protein sequences with hydrogen cyanide synthases from $P$. aeruginosa (PAOI) and P. fluorescens (PF-5). B. Sequence of HcnA C-terminal from PAOI and PF-5 aligned with B. cenocepacia putative proteins, BCAMOI268a, BCAM0669 and BCASOI64. Diamonds indicate a potential Fe-S binding site at 4 cysteine residues in HcnA. C. Sequence of the start of $\mathrm{HcnB}$ from PAOI and PF-5 aligned with B. cenocepacia putative proteins BCAM01269, BCAM0668 and BCASOI63. D. Sequence from the start of HcnC sequence from PAOI and PF-5 aligned with B. cenocepacia putative proteins BCAM0 1268, BCAM0667 and BCASOI 65. Arrows indicate eleven amino acid ADP-binding motif in HcnB and HcnC. 
Cyanide is produced by B. cenocepacia strains $\mathrm{J} 2315$ and K56-2 when grown on solid medium

Cyanide production by $P$. aeruginosa can also be assayed from cultures grown on solid media [24-26]. Therefore, $B$. cenocepacia was grown on LB agar medium and any cyanide given off during colonial growth trapped and assayed. Under these colonial growth conditions cyanide production was detected from both B. cenocepacia K56-2 and J2315. The concentrations of cyanide trapped were $8.9 \mathrm{mM}$ from K56-2 and $240 \mu \mathrm{M}$ from J2315, while from P. aeruginosa PAO1 it was $511 \mu \mathrm{M}$ (Fig. 2A). However, when normalised to the CFU count of the culture cyanide production from both $B$. cenocepacia strains was similar to that of $P$. aeruginosa (Fig. $2 \mathrm{~B}$ ). This demonstrated that $B$. cenocepacia strains were cyanogenic and that the levels of cyanide produced were comparable to those produced by P. aeruginosa.

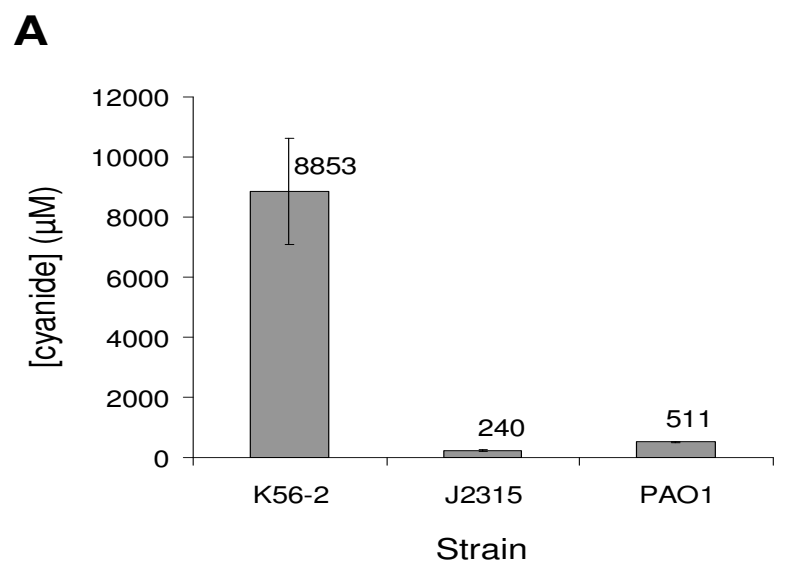

B

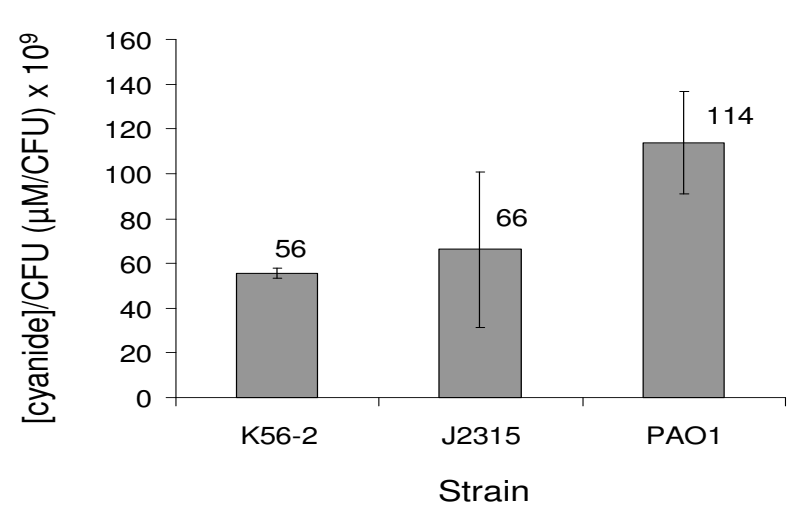

\section{Figure 2}

Cyanide production by $P$. aeruginosa and $B$. cenocepacia grown on solid media. A. Concentration of cyanide given off by plate grown culture and trapped in $\mathrm{NaOH}$. B. Concentration of trapped cyanide normalised to the CFU count for the plate. Values are the averages of 3 independent replicates with SE error bars.

\section{Cyanide is produced by all species of the Bcc when grown on solid media}

We next screened other members of the Bcc for cyanide production. A total of 34 strains with at least one representative from each species (genomovar) were assayed using the LB agar plate method (Table 1). Every strain tested was cyanogenic except for two B. dolosa strains, LMG18943 and LMG18944 (numbers 30 and 31 in Fig. $3)$. There was a greater than $2 \log$ variation $(\sim 60 \mu \mathrm{M}$ to 19 $\mathrm{mM}$ ) in the concentrations of cyanide trapped from the different Bcc species (Fig. 3A), and a greater than 5 log variation $\left(0.03 \mu \mathrm{M} / 10^{9} \mathrm{CFU}\right.$ to $\left.13000 \mu \mathrm{M} / 10^{9} \mathrm{CFU}\right)$ when the concentrations were normalised to the CFU counts obtained from the plates (Fig. 3B). In this assay a P. aeruginosa $\Delta$ hon strain produced no detectable cyanide [23]. The limited number of replicates for some strains meant that mean cyanide production could only be compared between B. cepacia, B. multivorans, B. cenocepacia and B. stabilis (nonparametric ANOVA, Kruskal - Wallis test). The mean normalised cyanide concentration for the B. stabilis strains $\left(2.2 \mu \mathrm{M} / 10^{9} \mathrm{CFU}\right)$ was significantly lower than the means for both the $B$. cepacia strains $\left(325 \mu \mathrm{M} / 10^{9}\right.$ $\mathrm{CFU})$ and $B$. cenocepacia strains (792 $\left.\mu \mathrm{M} / 10^{9} \mathrm{CFU}\right)$ (Dunn's multiple comparisons test, $\mathrm{p}<0.05$ for both), no other significant differences were seen in mean cyanide production between strains. All strains were also assayed for cyanide production in liquid culture, but like K56-2 and J2315 none of them tested positive.

In summary, all nine species in the Bcc are capable of producing cyanide when grown on solid media. The level of cyanide production in the Bcc appears to be strain rather than species specific.

\section{Is cyanide production in B. cenocepacia a biofilm specific phenotype?}

The finding that cyanide was not detected in liquid cultures of $B$. cenocepacia and other members of the Bcc complex, but was readily detected from plate grown cultures suggested that cyanide production in Bcc is a surface attached phenotype. Bcc can grow as a biofilm [10,27] and we were interested to test whether B. cenocepacia produces cyanide when growing surface-attached as a biofilm. We grew Bcc strains as biofilms on $6 \mathrm{~mm}$ diameter glass beads immersed in LB broth in a Petri dish, which provided a large surface area for growth. Biofilm growth was detected visually and by crystal violet staining $[10,27]$. Cyanide production was assessed by trapping cyanide given off from the biofilm culture in $4 \mathrm{M} \mathrm{NaOH}$. Control cultures comprised Petri dishes containing bacterial culture without glass beads. Figure 4A shows the data obtained for the $P$. aeruginosa PAO1. Cyanide was detected in the presence and absence of glass beads, consistent with $P$. aeruginosa being able to make HCN during 

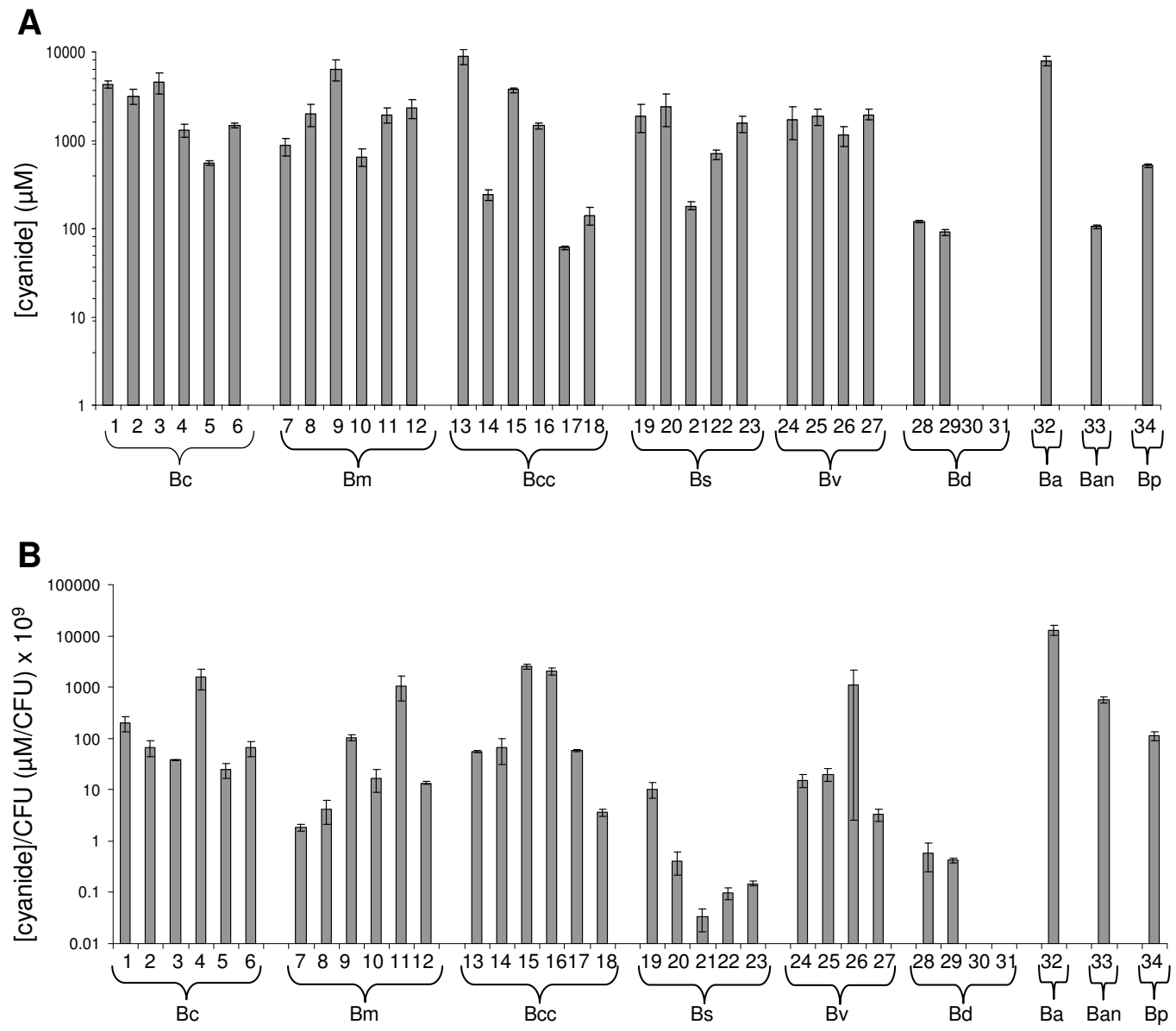

\section{Figure 3}

Cyanide production by members of the Bcc grown on solid media. Concentration of cyanide given off by plate grown cultures of Bcc strains. A. Concentration of cyanide trapped in $\mathrm{NaOH}$. B. Cyanide concentrations from A normalised to the CFU count for the plate. Values are the averages of 3 independent replicates with SE error bars. B.c., B. cepacia; B.m., B. multivorans; B.cc., B. cenocepacia;B.s., B. stabilis, B.v., B. vietnamensis; B.d., B. dolosa; B.a., B. ambifaria; B.an, B. anthina; B.p., B. pyrrocinia. The numbers under the axis correspond to stain names (for key to strain names see Table I).

planktonic growth, but after three days incubation cyanide was no longer detected (data not shown).

For the B. cenocepacia strains J2315 and K56-2 cyanide was only detected from the glass bead biofilm cultures and not from the control planktonic cultures (Fig. 4B and 4C). Cyanide was not detected until day 2 or day 3 for K56-2 and 2315 respectively. This is consistent with cyanide production resulting from biofilm growth, which takes time to establish on the glass bead surface.

Providing B. cenocepacia strains with a large surface area suitable for cell attachment/biofilm formation led to the detection of cyanide from the culture. This, together with the findings that Bcc strains produce detectable cyanide when grown on solid media but not in liquid culture, sug- gests that cyanide production in B. cenocepacia and other members of the Bcc is enhanced in surface attached cells and is a biofilm specific phenotype.

\section{Discussion}

In this paper we have demonstrated that B. cenocepacia and each of the eight other species of the Burkholderia cepacia complex can produce cyanide. This is the first time that any member of the Bcc has been reported to produce cyanide, although there are published reports of unsuccessful attempts to detect cyanide production by $B$. cepacia strains $[28,29]$. We can only surmise that strain or growth condition differences prevented the detection of cyanide production in these previous studies. Like $P$. aeruginosa, members of the Bcc are important pathogens of the $\mathrm{CF}$ lung $[7,30]$. Therefore, this finding is potentially signifi- 
Table I: Bacterial strains used in this study.

\begin{tabular}{|c|c|c|}
\hline Number in figure 3 & Strain & Description \\
\hline & P. aeruginosa & \\
\hline & PAOI & Wild-type, prototrophic strain \\
\hline & Burkholderia cepacia & \\
\hline I & LMG6993 & Environmental strain, soil \\
\hline 2 & LMG6988 & Clinical isolate, leg wound \\
\hline 3 & LMG6963 & Environmental isolate, soil \\
\hline 4 & LMG I 222 & Environmental isolate, onion \\
\hline 5 & LMG I882I & Clinical isolate, $C F$ lung \\
\hline \multirow[t]{2}{*}{6} & LMGI4095 & Clinical isolate, CF lung \\
\hline & Burkholderia multivorans & \\
\hline 7 & LMGI3010 & Clinical isolate, CF lung \\
\hline 8 & LMGI6660 & Clinical isolate, CF lung \\
\hline 9 & LMGI6665 & Clinical isolate, brain abscess \\
\hline 10 & LMGI7588 & Environmental isolate, soil \\
\hline 11 & LMG I 8822 & Clinical isolate, CF lung \\
\hline \multirow[t]{2}{*}{12} & LMG 8945 & Clinical isolate, CF lung \\
\hline & Burkholderia cenocepacia & \\
\hline 13 & $\mathrm{~K} 56-2$ & Clinical isolate, CF lung \\
\hline 14 & $\mathrm{~J} 2315$ & Clinical isolate, CF lung \\
\hline 15 & LMGI 6654 & Clinical isolate, CF lung \\
\hline 16 & LMGI6659 & Clinical isolate, $C F$ lung \\
\hline 17 & LMG 18830 & Clinical isolate, CF lung \\
\hline \multirow[t]{2}{*}{18} & LMGI8827 & Clinical isolate, CF lung \\
\hline & Burkholderia stabilis & \\
\hline 19 & LMG6997 & Clinical isolate, ear \\
\hline 20 & LMG7000 & Clinical isolate, blood \\
\hline 21 & LMGI429| & Clinical isolate, $C F$ lung \\
\hline 22 & LMGI4294 & Clinical isolate, $C F$ lung \\
\hline \multirow[t]{2}{*}{23} & LMG 18138 & Clinical isolate, CF lung \\
\hline & Burkholderia vietnamensis & \\
\hline 24 & LMGI8835 & Clinical isolate, CF lung \\
\hline 25 & LMG6998 & Clinical isolate, blood \\
\hline 26 & LMG 10928 & Environmental isolate, rice \\
\hline \multirow[t]{2}{*}{27} & LMGI6232 & Clinical isolate, CF lung \\
\hline & Burkholderia dolosa & \\
\hline 28 & LMGI894I & Clinical isolate, CF lung \\
\hline 29 & LMGI8942 & Clinical isolate, $C F$ lung \\
\hline 30 & LMG 18943 & Clinical isolate, CF lung \\
\hline \multirow[t]{2}{*}{31} & LMGI8944 & Clinical isolate, $C F$ lung \\
\hline & Burkholderia ambifaria & \\
\hline \multirow[t]{2}{*}{32} & LMG 19182 & Environmental isolate, pea rhizosphere \\
\hline & Burkholderia anthina & \\
\hline \multirow[t]{2}{*}{33} & LMG20983 & Clinical isolate, CF lung \\
\hline & Burkholderia pyrrocinia & \\
\hline 34 & LMGI4I9I & Environmental isolate, soil \\
\hline
\end{tabular}

All Bcc strains were obtained from the BCCM/LMG collection, University of Ghent, Ghent, Belgium.

cant in terms of CF lung infection, especially in light of our recent finding that cyanide accumulates in the lungs of $P$. aeruginosa-infected CF patients and is associated with a decline in lung function [13]. An interesting possibility is that Bcc infecting the CF lung makes cyanide, and that cyanide production provides a survival and/or colonisation advantage to CF-lung infecting bacteria.
Cyanide is a potent inhibitor of cellular respiration; it binds to and inhibits ferric iron containing enzymes including cytochrome $c$ oxidase. Bacterial cyanide production during lung infection would potentially damage local host tissue and possibly inhibit immune cell function. Thus, it is intriguing that the two major infecting bacteria of the CF lung are able to produce cyanide. 


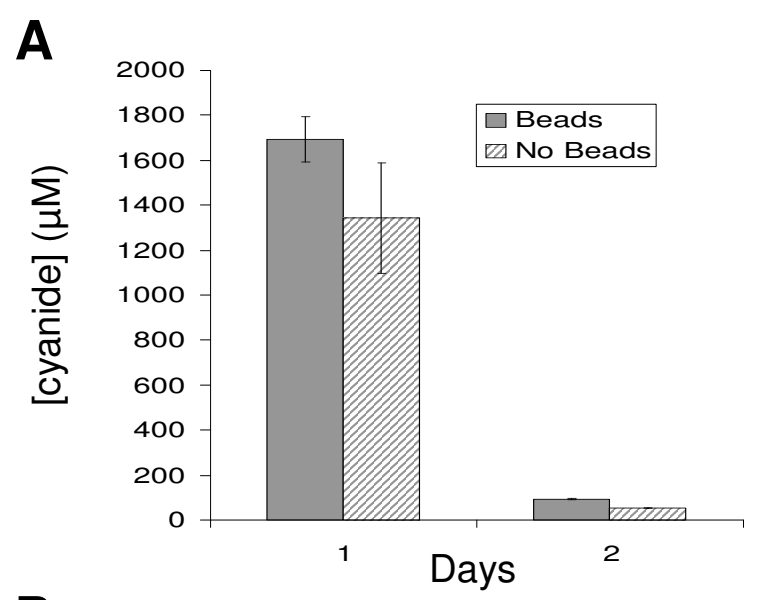

B
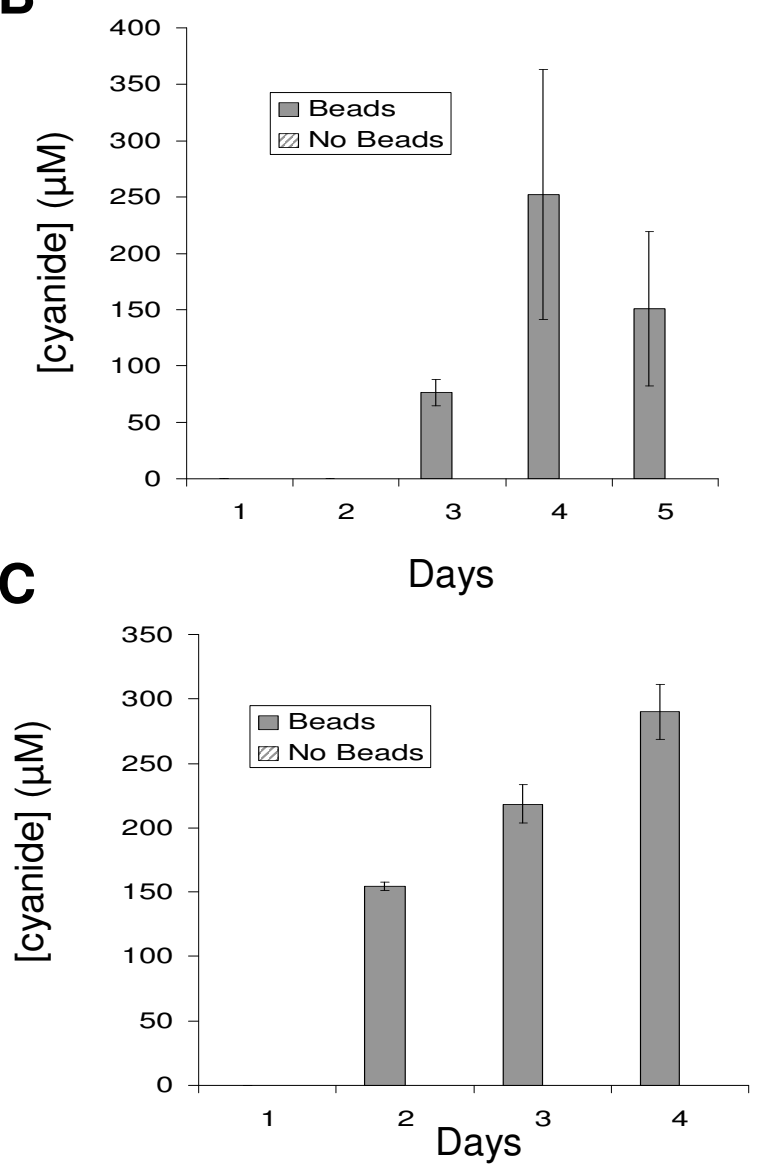

\footnotetext{
Figure 4

Cyanide production from glass bead "biofilm" model. Concentration of cyanide given off by glass bead biofilm cultures over 6 hours and trapped in I $\mathrm{ml} 4 \mathrm{M} \mathrm{NaOH}$. A., B. and $\mathbf{C}$. cyanide concentration trapped in $\mathrm{NaOH}$ for $P$. aeruginosa PAOI, B. cenocepacia 2315 and B. cenocepacia K56-2 respectively. Solid bars: with glass beads, diagonal striped bars: without glass beads. Measurements are averages of 3 independent replicates with SE error bars.
}

The variation in cyanide levels was strain rather than species specific, as is the pathogenicity of members of the Bcc in CF lung infection [4] and in the pathogenicity to C. elegans [31].

Cyanide production during planktonic growth in liquid culture was not detected for any members of the Bcc. It is possible that cyanide was produced but that it did not accumulate to a high enough level to be detected. However, in the plate cyanide assay the concentration of cyanide measured for many strains was significantly higher than that seen for $P$. aeruginosa PAO1, which does accumulate cyanide at readily detectable levels in liquid culture $[18,19,23]$. This suggests that Bcc strains are capable of producing cyanide at levels comparable to $P$. aeruginosa and that the absence of detectable cyanide in liquid cultures of the Bcc was not just because the strains are low level cyanide producers. The data presented in this paper is consistent with cyanide production in the Bcc being induced during colonial growth. The most common type of biofilm and the form usually investigated are those occurring between a solid/liquid interface, but the description can apply equally to the solid/air interface of colonial growth on an agar plate. It follows that cyanide production in the Bcc may be induced during biofilm growth. To test whether this occurs in B. cenocepacia, a solid/liquid interface biofilm model was used, which employed $6 \mathrm{~mm}$ glass beads in a Petri dish. B. cenocepacia produced cyanide in this model, and it took at least two days before cyanide was detected, suggesting that full establishment of the biofilm was necessary for cyanide production. These data support the view that cyanide production in $B$. cenocepacia is a biofilm specific phenotype. Work is now needed to determine the molecular mechanisms underlying cyanide production in the Bcc.

It is possible that cyanide production in biofilms is used to inhibit the growth of competing bacteria. Naturally occurring biofilms are usually made up of mixed populations of bacterial species; therefore it is likely that antimicrobial products will play a role in interspecies competition in biofilms. Indeed pyocyanin production by $P$. aeruginosa was found to inhibit B. cepacia's ability to form mixed biofilms with $P$. aeruginosa [27].

\section{Conclusion}

This paper reports that B. cenocepacia and the other members of Bcc produce cyanide. As such, they join a limited number of organisms that are capable of producing this potent inhibitor of aerobic respiration, but uniquely, to date they are the only bacteria described as being cyanogenic only during surface attached growth. 


\section{Methods}

Strains, growth media and culture conditions

The bacterial strains used in this study are listed in Table $1.1 \mathrm{ml}$ of an overnight starter culture grown in Luria-Bertani broth (10 g/l tryptone (Sigma), $5 \mathrm{~g} / \mathrm{l}$ yeast extract (Sigma), $5 \mathrm{~g} / \mathrm{l} \mathrm{NaCl}$ ), was used to inoculate $50 \mathrm{ml}$ of $\mathrm{LB}$ broth in a $250 \mathrm{ml}$ flask, which was then incubated in an orbital shaker at $200 \mathrm{rpm}$ at $37^{\circ} \mathrm{C}$. To look at the effects of oxygen on the ability of Bcc to produce cyanide during liquid growth, cultures were grown at three $\mathrm{O}_{2}$-transfer coefficient $\left(\mathrm{k}_{\mathrm{L}} \mathrm{a}\right)$ values: high $\left(87.4 \mathrm{~h}^{-1}\right)$, medium $\left(27.8 \mathrm{~h}^{-1}\right)$ and low $\left(11.5 \mathrm{~h}^{-1}\right)$ [21]. This was achieved by using identical $250 \mathrm{ml}$ flasks, shaking at $200 \mathrm{rpm}$, but altering the medium volume [21].

\section{Cyanide measurements}

An ELIT (ISM-146 CN) cyanide ion-selective micro-electrode (Lazar Research Laboratories, L.A., CA, USA) was used for measuring cyanide concentration in culture supernatants as described previously [23].

\section{Agar plate cyanide assay}

This method was adapted from [25]. LB agar plates were inoculated by spreading with $100 \mu \mathrm{l}$ of a 1:10 dilution of mid-log phase culture $\left(\mathrm{OD}_{600}=0.5\right)$ and incubated overnight at $37^{\circ} \mathrm{C}$. Plates, with lids removed, were then placed inside sterile $140 \mathrm{~mm}$ Petri dishes alongside a weigh boat containing $1 \mathrm{ml}$ of $4 \mathrm{M} \mathrm{NaOH}$. The $140 \mathrm{~mm}$ Petri dish was then sealed with Parafilm and incubated for 4 hours at $37^{\circ} \mathrm{C}$. The concentration of cyanide (given off by the culture) trapped in the $\mathrm{NaOH}$ was then measured and normalised to the CFU present on the plate. CFU counts were obtained by scraping the culture off the plate and suspending it in LB, dilutions were then plated out and colonies counted.

\section{Glass bead biofilm assay}

The glass bead biofilm apparatus consisted of a $90 \mathrm{~mm}$ Petri dish filled with $48 \mathrm{~g}$ of $6 \mathrm{~mm}$ glass beads (Fluka), which was sufficient to cover the bottom of the Petri dish in a single layer. It was inoculated with $12 \mathrm{ml}$ of a 1:10 dilution of mid-log phase culture $\left(\mathrm{OD}_{600}=0.5\right)$. After over night incubation at $37^{\circ} \mathrm{C}$ the culture medium was removed and replaced with fresh $\mathrm{LB}$ broth. The biofilm plates were then placed inside $140 \mathrm{~mm}$ Petri dishes alongside a weigh boat containing $1 \mathrm{ml}$ of $4 \mathrm{M} \mathrm{NaOH}$. The 140 $\mathrm{mm}$ Petri dishes were then sealed with Parafilm and incubated for 4 hours at $37^{\circ} \mathrm{C}$, and the concentration of cyanide trapped in the $\mathrm{NaOH}$ was measured. After cyanide measurement the culture media was removed from the biofilms and replaced with fresh LB broth and the biofilms were incubated at $37^{\circ} \mathrm{C}$ until the next day, when the process was repeated. Petri dishes without beads were included as controls; these were treated in exactly the same way as the glass bead biofilm plates

\section{Authors' contributions}

BR participated in the design and co-ordination of the study, carried out plate and liquid culture cyanide assays, conceived and developed the glass bead biofilm model, carried out the biofilm cyanide assays, carried out the statistical analysis and drafted the paper. XL carried out plate cyanide assays. SH carried out plate cyanide assays. JZ carried out the bioinformatics/sequence alignments and helped draft the manuscript. HW conceived of the study, participated in its design and co-ordination and wrote the final draft of the manuscript.

\section{Acknowledgements}

This work was supported by the UK Biotechnology and Biological Sciences Research Council through a studentship to BR.

\section{References}

I. Coenye T, Vandamme P: Diversity and significance of Burkholderia species occupying diverse ecological niches. Environmental Microbiology 2003, 5(9):719-729.

2. Vandamme P, Govan J, LiPuma J: Diversity and role of Burkholderia spp. In Burkholderia Molecular Microbiology and Genomics Edited by: Coenye T, Vandamme P. Horizon Bioscience; 2007.

3. Chiarini L, Bevivino A, Dalmastri C, Tabacchioni S, Visca P: Burkholderia cepacia complex species: health hazards and biotechnological potential. Trends in Microbiology 2006, 14(6):277-286.

4. Mahenthiralingam E, Urban TA, Goldberg JB: The multifarious, multireplicon Burkholderia cepacia complex. Nature Reviews Microbiology 2005, 3(2): 144-156.

5. Vandamme P, Holmes B, Vancanneyt M, Coenye T, Hoste B, Coopman R, Revets H, Lauwers S, Gillis M, Kersters K, Govan JRW: Occurrence of multiple genomovars of Burkholderia cepacia in cystic fibrosis patients and proposal of Burkholderia multivorans sp. nov. International Journal of Systematic Bacteriology 1997, 47(4): I I 88- 1200.

6. Govan JRW, Deretic V: Microbial pathogenesis in cystic fibrosis: mucoid Pseudomonas aeruginosa and Burkholderia cepacia. Microbiological Reviews 1996, 60(3):539-574.

7. Mahenthiralingam E, Baldwin A, Vandamme P: Burkholderia cepacia complex infection in patients with cystic fibrosis. Journal of Medical Microbiology 2002, 5I(7):533-538.

8. Banerjee D, Stableforth D: The treatment of respiratory Pseudomonas infection in cystic fibrosis - What drug and which way? Drugs 2000, 60(5): 1053-1064.

9. Isles A, Maclusky I, Corey M, Gold R, Prober C, Fleming P, Levison H: Pseudomonas Cepacia Infection in Cystic-Fibrosis - an Emerging Problem. Journal of Pediatrics 1984, 104(2):206-210.

10. Conway BAD, Venu V, Speert DP: Biofilm formation and acyl homoserine lactone production in the Burkholderia cepacia complex. Journal of Bacteriology 2002, I84(20):5678-5685.

II. Eberl L: Quorum sensing in the genus Burkholderia. International Journal of Medical Microbiology 2006, 296(2-3): I03-II0.

12. Subsin B, Chambers CE, Visser MB, Sokol PA: Identification of genes regulated by the CepIR quorum-sensing system in Burkholderia cenocepacia by high-throughput screening of a random promoter library. Journal of Bacteriology 2007, 189(3):968-979.

13. Ryall B, Davies JC, Wilson R, Shoemark A, Williams HD: Cyanide accumulates in the sputum of Pseudomonas aeruginosa infected cystic fibrosis and non-cystic fibrosis patients and is associated with impaired lung function. European Respiratory Journal 2008, Epub ahead of print:.

14. Adjei MD, Ohta Y: Isolation and characterization of a cyanideutilizing Burkholderia cepacia strain. World Journal of Microbiology \& Biotechnology 1999, 15(6):699-704.

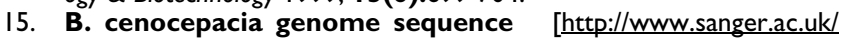
Projects/B cenocepacia/]

16. P. aeruginosa genome sequence [http://www.pseu
P. domonas.com] 
17. Laville J, Blumer C, Von Schroetter C, Gaia V, Defago G, Keel C, Haas $D$ : Characterization of the hcnABC gene cluster encoding hydrogen cyanide synthase and anaerobic regulation by ANR in the strictly aerobic biocontrol agent Pseudomonas fluorescens CHAO. Journal of Bacteriology 1998, I 80(12):3।87-3196.

18. Zlosnik JEA, Tavankar GR, Bundy JG, Mossialos D, O'Toole R, Williams HD: Investigation of the physiological relationship between the cyanide-insensitive oxidase and cyanide production in Pseudomonas aeruginosa. Microbiology 2006 I52:1407-1415

19. Blumer C, Haas D: Mechanism, regulation, and ecological role of bacterial cyanide biosynthesis. Archives of Microbiology 2000, 173(3): 170-177.

20. Castric PA: Glycine metabolism by Pseudomonas aeruginosa hydrogen cyanide biosynthesis. Journal of Bacteriology 1977, | 30(2):826-83|.

21. Cooper M, Tavankar GR, Williams HD: Regulation of expression of the cyanide-insensitive terminal oxidase in Pseudomonas aeruginosa. Microbiology 2003, 1 49: I 275- 1284.

22. Williams HD, Zlosnik JEA, Ryall B: Oxygen, cyanide and energy generation in the cystic fibrosis pathogen Pseudomonas aeruginosa. Advances in Microbial Physiology, Vol 52 2007, 52:I-7I.

23. Zlosnik JEA, Williams HD: Methods for assaying cyanide in bacterial culture supernatant. Letters in Applied Microbiology 2004, 38(5):360-365.

24. Carterson AJ, Morici LA, Jackson DW, Frisk A, Lizewski SE, Jupiter R Simpson K, Kunz DA, Davis SH, Schurr JR, Hassett DJ, Schurr MJ: The transcriptional regulator AlgR controls cyanide production in Pseudomonas aeruginosa. Journal of Bacteriology 2004, I 86(20):6837-6844.

25. Gallagher LA, Manoil C: Pseudomonas aeruginosa PAOI kills Caenorhabditis elegans by cyanide poisoning. Journal of Bacteriology 200I, I83(2I):6207-62I4.

26. Lizewski SE, Schurr JR, Jackson DW, Frisk A, Carterson AJ, Schurr MJ: Identification of AlgR-regulated genes in Pseudomonas aeruginosa by use of microarray analysis. Journal of Bacteriology 2004, I 86( I 7):5672-5684.

27. Tomlin KL, Coll OP, Ceri H: Interspecies biofilms of Pseudomonas aeruginosa and Burkholderia cepacia. Canadian Journal of Microbiology 200 I, 47( I 0):949-954.

28. Bevivino A, Tabacchioni S, Chiarini L, Carusi MV, Delgallo M, Visca P: Phenotypic comparison between rhizosphere and clinical isolates of Burkholderia cepacia. Microbiology 1994 I 40:1069-1077.

29. Kothe M, Antl M, Huber B, Stoecker K, Ebrecht D, Steinmetz I, Eber $\mathrm{L}$ : Killing of Caenorhabditis elegans by Burkholderia cepacia is controlled by the cep quorum-sensing system. Cellular Microbiology 2003, 5(5):343-35I.

30. Lyczak JB, Cannon CL, Pier GB: Lung infections associated with cystic fibrosis. Clinical Microbiology Reviews 2002, I 5(2):194-222.

31. Cardona ST, Wopperer J, Eberl L, Valvano MA: Diverse pathogenicity of Burkholderia cepacia complex strains in the Caenorhabditis elegans host model. FEMS Microbiology Letters 2005, 250(I):97-104.

Publish with BioMed Central and every scientist can read your work free of charge

"BioMed Central will be the most significant development for disseminating the results of biomedical research in our lifetime. "

Sir Paul Nurse, Cancer Research UK

Your research papers will be:

- available free of charge to the entire biomedical community

- peer reviewed and published immediately upon acceptance

- cited in PubMed and archived on PubMed Central

- yours - you keep the copyright
BioMedcentral 